\title{
TESTE E DIFUSÃO DE SISTEMAS AGROECOLÓGICOS DE MELHORAMENTO DO SOLO
}

\author{
Cíntia Schelbauer ${ }^{1}$ \\ Maiara Leonel Pereira ${ }^{2}$ \\ Kamilly Amorin Gracia ${ }^{3}$ \\ Marcelo Venturi ${ }^{4}$ \\ Paulo Emilio Lovato ${ }^{5}$ \\ Jucinei José Comin ${ }^{6}$
}

\section{RESUMO}

Neste artigo são apresentados resultados de um trabalho de extensão e pesquisa participativa, realizado no ano de 2007, em propriedades agrícolas nas quais se testaram, em diferentes regiões de Santa Catarina, sistemas agroecológicos de melhoramento do solo em parceria com agricultores. Os agricultores foram habilitados a medir os melhores tratamentos no controle de plantas espontâneas, pragas e doenças, a qualidade do solo e a produtividade da cultura econômica. Verificou-se que muitos agricultores percebem a interação entre determinadas características do solo e as influências que umas provocam nas outras. Entre os pontos mais abordados pelos agricultores, destacam-se aqueles relacionados à qualidade do solo, o que demonstra o entendimento das características do solo benéficas às suas funções e que estão diretamente ligadas à produção. Quanto à sustentabilidade, pouca ênfase foi dada pelos agricultores, evidenciando deficiências no conhecimento da produção sustentável e seus benefícios.

Palavras-chave: Qualidade do solo. Sustentabilidade. Biodiversidade.

\footnotetext{
${ }^{1}$ Engenheira Agrônoma - cynthya_sc@yahoo.com.br

2 Graduanda do Curso de Agronomia da UFSC - maiaraleonel@yahoo.com.br

${ }^{3}$ Engenheira Agrônoma - kamillygarcia@hotmail.com

${ }^{4}$ Mestrando do Programa de Pós-Graduação em Agroecossistemas da UFSC - vento_mar@hotmail.com

${ }^{5}$ Professor Associado II do Departamento de Engenharia Rural da UFSC - plovato@mbox1.ufsc.br

${ }^{6}$ Professor Associado II do Departamento de Engenharia Rural da UFSC - jcomin@cca.ufsc.br
} 


\title{
RESEARCH AND EXTENSION OF AGROECOLOGICAL SYSTEMS FOR SOIL IMPROVEMENT
}

\begin{abstract}
This study shows results of a research done in little farms of Santa Catarina state, Brazil, where it was tested different management systems to the improvement of soil attributes. To develop this study, farmers were trained to measure the best treatments of weed, insect and diseases control, as well as soil quality (e.g. root development, cover crops, soil compaction) and corn and bean production. Farmers noted the interaction among some soil attributes and detached those related to soil quality as the most linked to plant production. As related to sustainability of the system, little emphasis was given by farmers, suggesting deficits in their knowledge about the benefits of sustainable agriculture production.
\end{abstract}

Keywords: Soil quality. Sustainability. Biodiversity.

\section{INTRODUÇÃo}

O uso do solo através do sistema convencional de manejo com seu intenso revolvimento, com a finalidade de incorporar adubos, calcário ou, ainda, controlar ou suprimir as plantas espontâneas e a busca por elevados rendimentos das culturas agrícolas tem causado erosão, compactação, redução na capacidade do solo de armazenar água, diminuição dos teores de matéria orgânica e da atividade biológica no solo, deixando-o menos fértil e menos apto à agricultura. As operações de preparo do solo também acarretam aumento de custos de produção, como horas-máquina, combustível, manutenção e aquisição e aplicação de agrotóxicos.

Essa exploração intensiva dos recursos naturais, com o objetivo de obter maior produtividade, e o manejo inadequado dos solos agrícolas, tem proporcionado aumento significativo de terras degradadas, comprometendo a qualidade do solo, entendida como a capacidade de o solo funcionar, dentro dos limites dos ecossistemas e das terras agrícolas, sustentar a produtividade biológica, manter a qualidade do ambiente e manter ou melhorar a saúde humana, animal e das plantas (DORAN; PARKIN, 1994).

O principal problema que leva o homem a cometer tais erros é a falta de conhecimento a respeito do ambiente em que habita e conduz seus sistemas produtivos. Felizmente, existem agricultores que convivem de um modo diferente com a propriedade e os seus componentes e 
têm a percepção das interações que ocorrem entre plantas, animais, solo, ar, água, o que é muito importante para entender alguns ciclos, intensificá-los e minimizar os desperdícios. A percepção define-se como o processo de organizar e interpretar dados sensoriais recebidos (sensações) para desenvolvermos a consciência do ambiente que nos cerca e de nós mesmos. A percepção, portanto, implica interpretação (DAVIDOFF, 1983 apud VALLE; COSTA, 2008).

A compreensão dessas interações conduz à sustentabilidade, de forma que a propriedade possa ser manejada para utilizar todos os recursos possíveis e interagir diversas atividades, adotar sistemas de proteção do solo e maximizar a biodiversidade.

Para permitir uma melhor interação do agricultor com o ambiente é necessário desenvolver em conjunto parâmetros de percepção rápida; que o agricultor use-os no seu dia a dia e, com eles, possa compreender métodos bons e ruins de manejo dos agroecossistemas dos quais se utiliza. Assim, passará a melhorar os métodos de cultivo, identificados através das melhores colheitas em termos de qualidade e quantidade, bem como a preservação do meio.

Stocking e Murnaghan (2001) levantam três vantagens principais de realizar um diagnóstico participativo de avaliação do solo: a) o agricultor percebe sinais reais de degradação e de perda de qualidade do solo. Esta percepção é muito mais importante do que complexas análises laboratoriais; b) os resultados de diagnóstico no campo tendem a integrar uma série de processos complexos de degradação, ao contrário do que ocorre com o modelo cartesiano tradicional que secciona os processos naturais para estudá-los separadamente e acaba perdendo informações importantes; c) o agricultor aprende com sua experiência a ser prático e a dar atenção apenas aos aspectos importantes.

Por outro lado, os mesmos autores também alertam para as desvantagens da metodologia, como a exatidão das observações que geralmente não é muito grande, e o fato da percepção da degradação ou da qualidade do solo ser um processo integrado entre diversos elementos, o que torna difícil extrapolar seus resultados para outros ecossistemas e as comparações com outros agricultores.

Os objetivos do presente trabalho foram: testar, em diferentes regiões de Santa Catarina, sistemas de melhoramento do solo, definidos a partir de resultados de pesquisas realizadas pela Universidade Federal de Santa Catarina - UFSC e pela Empresa de Pesquisa Agropecuária e Extensão Rural de Santa Catarina - EPAGRI, definindo, através da opinião dos agricultores, qual a melhor combinação de espécies nos sistemas de melhoramento do solo em diversas regiões de Santa Catarina, para realização de plantio direto sem o uso de 
herbicidas e outros agrotóxicos. Assim, foram estabelecidas aproximadamente 50 unidades de estudo (demonstrativas/experimentais) em propriedades agrícolas no Estado, através de projeto de extensão e pesquisa participativa, no qual agricultores foram habilitados a medir, utilizando indicadores específicos, os melhores tratamentos no controle de plantas espontâneas, pragas e doenças, qualidade do solo e produtividade da cultura econômica. As unidades de estudo das propriedades agrícolas também foram utilizadas como objeto de discussão e formação de técnicos, estudantes e agricultores e foram realizados dias de campo com agricultores, estudantes, técnicos e dirigentes municipais para validação e divulgação de sistemas agroecológicos de melhoramento do solo.

\section{MATERIAL E MÉTODOS}

Algumas atividades de campo, das quais as bolsistas do projeto participaram, foram realizadas nas propriedades onde instalaram-se as parcelas experimentais, em municípios previamente escolhidos por técnicos da EPAGRI. Ao todo, foram 70 parcelas distribuídas em 25 municípios. Destas, oito parcelas foram instaladas no Centro de Desenvolvimento do Jovem Rural de Lauro Muller - CEDEJOR, uma em aldeia indígena em Imaruí e seis em Passos Maia, no assentamento do Movimento Sem Terra - MST. Os municípios e o número de agricultores participantes por unidade estão descritos no quadro 1.

\begin{tabular}{|l|c|}
\hline \multicolumn{1}{|c|}{ Município } & $\mathbf{N}^{\mathbf{0}}$ de agricultores \\
\hline Anita Garibaldi & 2 \\
\hline Brunópolis & 2 \\
\hline Campo Belo do Sul & 1 \\
\hline Celso Ramos & 3 \\
\hline Chapecó & 5 \\
\hline Garuva & 4 \\
\hline Guaraciaba & 2 \\
\hline Guatambu & 3 \\
\hline Ibiam & 2 \\
\hline Imaruí & 1 \\
\hline Lontras & 3 \\
\hline Mondai & 2 \\
\hline Monte Carlo & 3 \\
\hline Morro da Fumaça & 5 \\
\hline Nova itaberaba & 2 \\
\hline Passos Maia & 6 \\
\hline Planalto Alegre & 1 \\
\hline
\end{tabular}




\begin{tabular}{|l|c|} 
Presidente Nereu & 4 \\
\hline Rancho Queimado & 2 \\
\hline Santa Terezinha & 2 \\
\hline Rio dos Cedros & 1 \\
\hline Santa Terezinha do Progresso & 3 \\
\hline São Martinho & 1 \\
\hline Lauro Muller / CEDEJOR & 8 \\
\hline Zórtea & 2 \\
\hline Total $=25$ & total $=70$ \\
\hline
\end{tabular}

Quadro 1: Municípios participantes do projeto e número de agricultores por município. Fonte: Garcia (2007)

Devido ao grande número de agricultores que participavam do projeto, as visitas e os dias de campo foram divididos em regiões.

Em cada propriedade foi instalada uma lavoura experimental de aproximadamente 800 $\mathrm{m}^{2}$, dividida em quatro tratamentos. Os tratamentos consistiram de diferentes proporções de três espécies de plantas de cobertura (ervilhaca, nabo forrageiro e centeio) conforme demonstrado no quadro 2.

\begin{tabular}{|l|}
\hline A: $1 / 3$ de centeio $+1 / 3$ de ervilhaca $+1 / 3$ de nabo. \\
\hline B: $1 / 2$ de centeio $+1 / 4$ de ervilhaca $+1 / 4$ de nabo. \\
\hline C: $1 / 4$ de centeio $+1 / 2$ de ervilhaca $+1 / 4$ de nabo. \\
\hline D: $1 / 4$ de centeio $+1 / 4$ de ervilhaca $+1 / 2$ de nabo. \\
\hline
\end{tabular}

Quadro 2: Proporção das culturas de coberturas nos quatro tratamentos.

Fonte: elaborado pelos autores, 2007.

A semeadura dessas espécies para cobertura do solo no período de outono e inverno foi efetuada por sobressemeadura. Previamente à semeadura das espécies de verão, as plantas de cobertura foram manejadas com rolo-faca. A semeadura das culturas econômicas de verão foi realizada com equipamentos manuais ou tratorizados para plantio direto e o sistema adotado foi sem o uso de herbicidas.

É premente a necessidade de adoção de práticas de controle integrado de plantas espontâneas que transformem o uso profilático de agrotóxicos em uso seletivo, permitindo, assim, a diminuição da dependência de insumos externos e a maximização do uso de controles físicos e biológicos de espontâneas, doenças e pragas, a fim de diminuir o impacto ambiental dos cultivos (DOYLE, 1997). Algumas culturas de cobertura de inverno podem auxiliar no controle de espontâneas, diminuindo a população ou reduzindo o potencial de competição por água, luz, nutrientes e solo destas com o cultivo (KOHLI et al., 2006). 
As culturas foram implantadas em monocultivo ou policultivo, conforme a ilustração 1. No inverno todas as unidades experimentais foram semeadas com as diferentes proporções de centeio, ervilhaca e nabo forrageiro.

\section{Verão:}

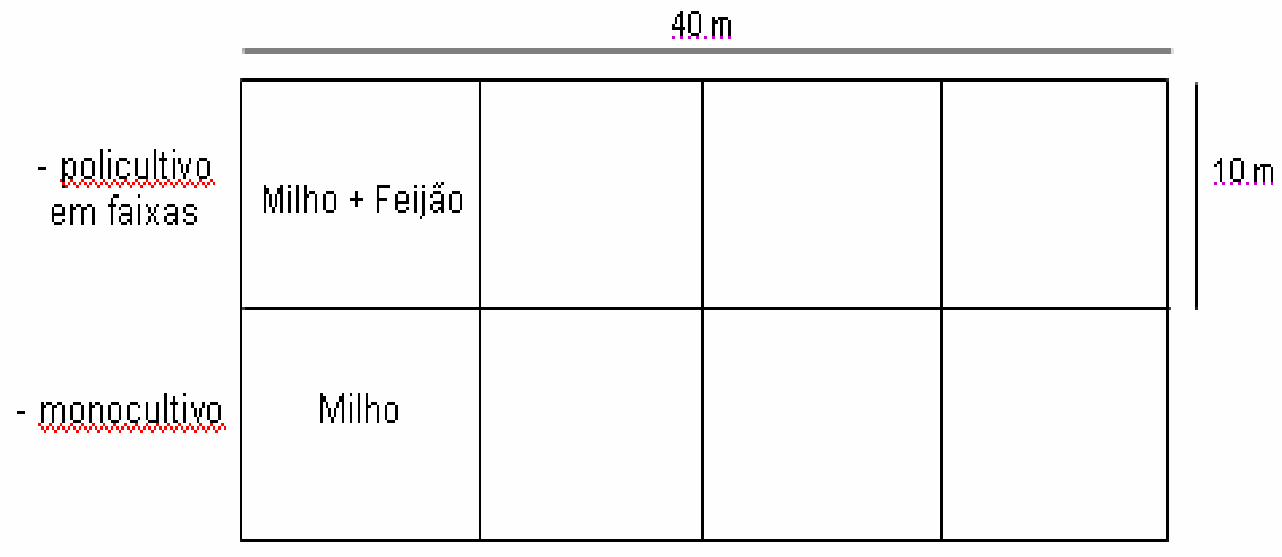

Ilustração 1: Croqui das unidades experimentais para as culturas econômicas de verão. Fonte: elaborado pelos autores, 2007.

As visitas para capacitações dos agricultores foram conduzidas pelos mestrandos e/ou professores e tiveram a participação dos alunos de graduação bolsistas e/ou voluntários do projeto. As capacitações iniciaram a partir da implantação das lavouras de outono-inverno e ocorreram ao longo do ciclo dessas culturas. Nelas foram trabalhados históricos, princípios e vantagens do sistema de plantio direto. Também foi apresentada a metodologia dos indicadores de qualidade do solo e de sustentabilidade.

Esta metodologia consiste primeiramente na identificação de sustentabilidade e qualidade do solo, e, em seguida, na seleção dos indicadores que servirão para caracterizar, avaliar e monitorar a qualidade do solo bem como a sustentabilidade do meio. Finalmente serão dadas notas aos indicadores pelos agricultores, gerados gráficos do tipo radar (NICHOLLS et al., 2004) e os resultados são discutidos.

Após as primeiras visitas, foram distribuídos três questionários aos agricultores. Esses eram compostos das mesmas questões, mas foram respondidos em três momentos diferentes para permitir a avaliação da evolução dos sistemas produtivos. O primeiro questionário foi preenchido no momento da implantação das culturas de cobertura de inverno, o segundo, 30 dias após a emergência das plantas de cobertura, e o terceiro, no momento do manejo das culturas de cobertura de inverno com o rolo-faca. 
Em cada um desses momentos foram trabalhados os seguintes indicadores:

a) cobertura e espessura de palhada; cobertura de plantas espontâneas; estrutura do solo; quantidade de matéria orgânica; atividade biológica; umidade do solo; resistência a penetração e atividade microbiológica;

b) cobertura de plantas espontâneas e cobertura de plantas cultivadas. Nestas avaliações não foi solicitado aos agricultores para dar nota, mas para jogar um quadro de madeira de 50 x $50 \mathrm{~cm}$ duas vezes dentro de cada parcela e realizar uma estimativa do percentual de cada quadro ocupado por plantas espontâneas, por plantas de cobertura e por solo exposto;

c) cobertura e espessura de palhada; cobertura de plantas espontâneas; cobertura de plantas cultivadas; desenvolvimento das plantas cultivadas (cobertura de inverno); estrutura do solo; quantidade de matéria orgânica; atividade microbiológica; umidade do solo; resistência a penetração e atividade microbiológica.

Com as notas atribuídas a esses parâmetros foram construídos os gráficos tipo radar para permitir uma melhor representação das notas. O gráfico consiste em um círculo dividido em várias partes através de vários diâmetros. As notas são, então, assinaladas no intervalo de zero a dez, no qual o zero é o centro do círculo e dez é o ponto em que a linha encontra a circunferência, conforme exemplificado na ilustração 2.

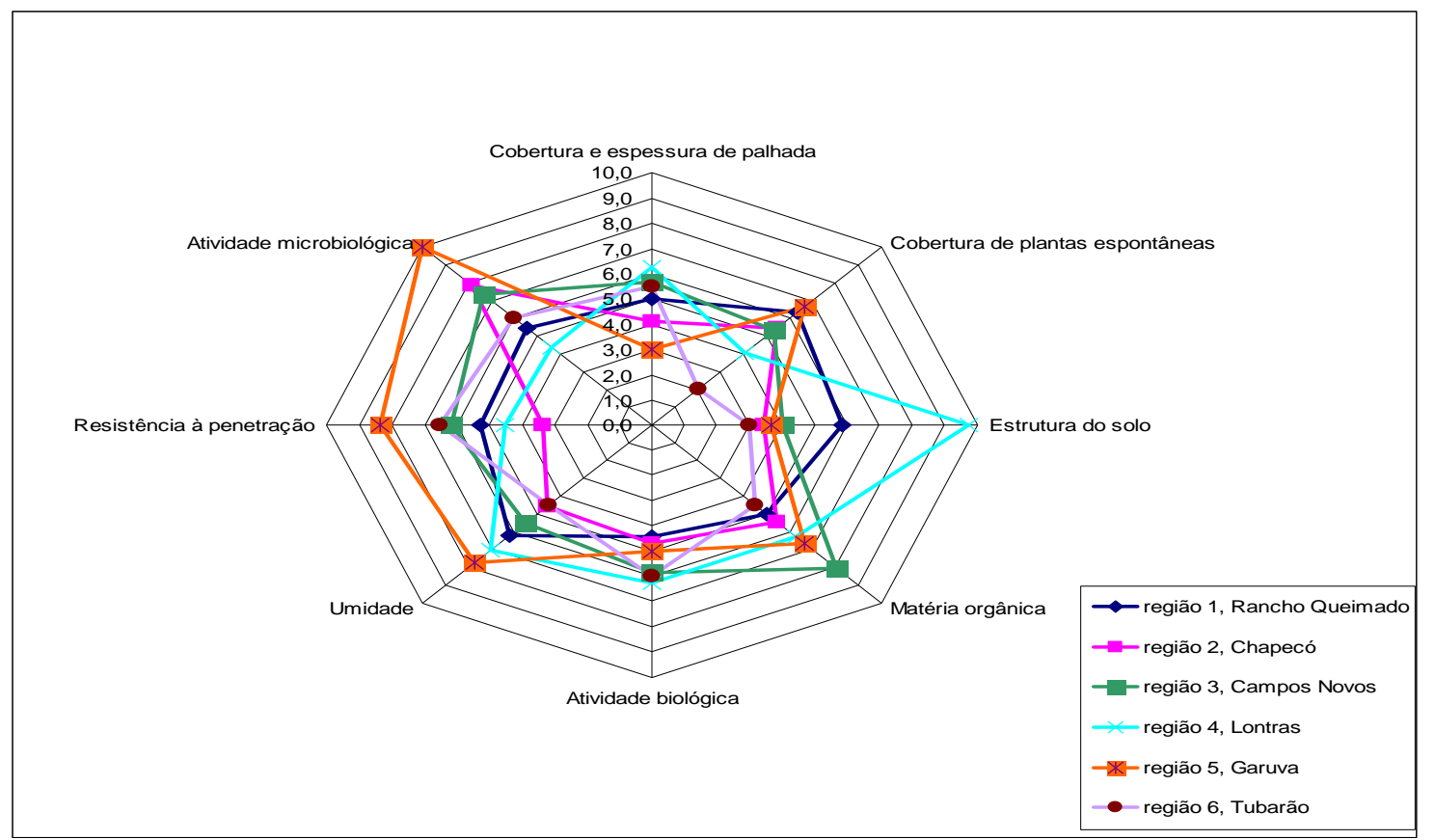

Ilustração 2: Representação gráfica dos valores médios atribuídos a cada indicador de qualidade do solo em seis regiões de Santa Catarina.

Fonte: Garcia, 2007. 


\section{RESULTADOS E ANÁLISE}

A partir da análise dos questionários se percebeu que a maioria dos agricultores já fazia uso do sistema de plantio direto, o que não reflete a realidade de nosso Estado, pois há uma grande quantidade de agricultores que resistem em abandonar os métodos de cultivo convencional. Os indicadores levantados pelos agricultores para avaliar a qualidade dos seus sistemas produtivos nas diferentes regiões do estudo estão representados no quadro 3.

\begin{tabular}{|c|c|c|c|c|c|c|}
\hline \multirow[t]{2}{*}{ INDICADOR } & \multicolumn{6}{|c|}{ CAPACITAÇÕES } \\
\hline & Rancho Q. & Tubarão & Chapecó & Garuva & Lontras & $\begin{array}{c}\text { Campos } \\
\text { Novos }\end{array}$ \\
\hline Terra fofa/solta/macia & & & $\mathrm{X}$ & $\mathrm{X}$ & $\mathrm{X}$ & \\
\hline Cobertura morta/palhada & $\mathrm{X}$ & $\mathrm{X}$ & $\mathrm{X}$ & $\mathrm{X}$ & $\mathrm{X}$ & $\mathrm{X}$ \\
\hline Cor do solo & $\mathrm{X}$ & $\mathrm{X}$ & $\mathrm{X}$ & & $\mathrm{X}$ & $\mathrm{X}$ \\
\hline Erosão & $\mathrm{X}$ & $\mathrm{X}$ & $\mathrm{X}$ & $\mathrm{X}$ & & $\mathrm{X}$ \\
\hline Textura & $\mathrm{X}$ & $\mathrm{X}$ & $\mathrm{X}$ & & $\mathrm{X}$ & \\
\hline Retenção de água e O2 & & & $\mathrm{X}$ & $\mathrm{X}$ & & $\mathrm{X}$ \\
\hline Organismos & & $\mathrm{X}$ & $\mathrm{X}$ & & $\mathrm{X}$ & $\mathrm{X}$ \\
\hline Matéria orgânica & $\mathrm{X}$ & $\mathrm{X}$ & $\mathrm{X}$ & $\mathrm{X}$ & $\mathrm{X}$ & $\mathrm{X}$ \\
\hline Cheiro & $\mathrm{X}$ & & $\mathrm{X}$ & & & $\mathrm{X}$ \\
\hline Compactação & $\mathrm{X}$ & $\mathrm{X}$ & $\mathrm{X}$ & & $\mathrm{X}$ & $\mathrm{X}$ \\
\hline Profundidade & & & $\mathrm{X}$ & $\mathrm{X}$ & $\mathrm{X}$ & $\mathrm{X}$ \\
\hline Tipo de solo & & & $\mathrm{X}$ & & & \\
\hline Umidade & $\mathrm{X}$ & & & $\mathrm{X}$ & $\mathrm{X}$ & $\mathrm{X}$ \\
\hline Pedregosidade & & $\mathrm{X}$ & & & & $\mathrm{X}$ \\
\hline Resistência à seca & & & & & & $\mathrm{X}$ \\
\hline Insetos & $\mathrm{X}$ & & & $\mathrm{X}$ & & $\mathrm{X}$ \\
\hline Microorganismos & & & & & & $\mathrm{X}$ \\
\hline Estrutura & & & & & & $\mathrm{X}$ \\
\hline Infiltração & & $\mathrm{X}$ & & & & $\mathrm{X}$ \\
\hline Análise & & & & $\mathrm{X}$ & $\mathrm{X}$ & \\
\hline Camadas do solo & & & & $\mathrm{X}$ & $\mathrm{X}$ & \\
\hline Temperatura & & & & & $\mathrm{X}$ & \\
\hline Minhocas & & & & & $\mathrm{X}$ & \\
\hline Luminosidade & & & & & $\mathrm{X}$ & \\
\hline Material de origem & & & & & $\mathrm{X}$ & \\
\hline Fertilidade & & $\mathrm{X}$ & & & & \\
\hline Estrutura da bosta & $\mathrm{X}$ & & & & & \\
\hline Desenvolvimento planta & & $\mathrm{X}$ & $\mathrm{X}$ & $\mathrm{X}$ & $\mathrm{X}$ & \\
\hline Diversidade de espontâneas & $\mathrm{X}$ & $\mathrm{X}$ & $\mathrm{X}$ & & $\mathrm{X}$ & $\mathrm{X}$ \\
\hline Cor das plantas & & $\mathrm{X}$ & $\mathrm{X}$ & & & \\
\hline Produtividade & & $\mathrm{X}$ & $\mathrm{X}$ & $\mathrm{X}$ & $\mathrm{X}$ & $\mathrm{X}$ \\
\hline Doenças & & $\mathrm{X}$ & $\mathrm{X}$ & $\mathrm{X}$ & $\mathrm{X}$ & $\mathrm{X}$ \\
\hline Pragas & & & $\mathrm{X}$ & $\mathrm{X}$ & $\mathrm{X}$ & $\mathrm{X}$ \\
\hline Cobertura verde & & & $\mathrm{X}$ & $\mathrm{X}$ & & $\mathrm{X}$ \\
\hline Vigor & & & & $\mathrm{X}$ & & $\mathrm{X}$ \\
\hline Plantas indicadoras & $\mathrm{X}$ & $\mathrm{X}$ & & $\mathrm{X}$ & $\mathrm{X}$ & $\mathrm{X}$ \\
\hline
\end{tabular}




\begin{tabular}{|l|c|c|c|c|c|c|}
\hline Quantidade de espontâneas & & & & & & X \\
\hline Competição entre plantas & $\mathrm{X}$ & & & & & \\
\hline Histórico de uso & & $\mathrm{X}$ & $\mathrm{X}$ & & & \\
\hline Disponibilidade de água & & & & $\mathrm{X}$ & & \\
\hline Relevo & $\mathrm{X}$ & $\mathrm{X}$ & & $\mathrm{X}$ & & \\
\hline Rotação de cultura & & & & $\mathrm{X}$ & & \\
\hline Produtos químicos & & & & & $\mathrm{X}$ & \\
\hline Localização no relevo & & & & & $\mathrm{X}$ & \\
\hline Proteção fontes água & & & & & $\mathrm{X}$ & \\
\hline Cobiça do vizinho & & & & $\mathrm{X}$ & & \\
\hline Clima (relação a região) & & $\mathrm{X}$ & & & & \\
\hline
\end{tabular}

Quadro 3: Indicadores de qualidade do solo propostos pelos agricultores para avaliar a qualidade de seus sistemas produtivos em diferentes regiões de Santa Catarina.

Fonte: Garcia (2007).

A análise dos questionários respondidos pelos agricultores e os indicadores de qualidade do solo por eles escolhidos demonstrou que muitos agricultores percebem a interação entre determinadas características do solo e as influências que umas provocam nas outras (Garcia, 2007). Como exemplo, podemos citar alguns parâmetros levantados nas capacitações, como a capacidade do solo em reter água, a resistência à seca, a infiltração de água no solo, e os indicadores relacionados à compactação e à textura, demonstrando que entendem a necessidade de manter o solo úmido e descompactado.

Constatou-se também que a diversidade de microrganismos no solo é vista como um ponto importante pelos agricultores, pois evidencia que há um meio favorável ao seu desenvolvimento, e este meio favorável representa equilíbrio de nutrientes e umidade. Para Zilli et al. (2003), o uso da diversidade microbiana como indicador de qualidade do solo vem tendo um avanço muito importante. Isso porque tem se tornado consenso que a diversidade microbiana possui importantes vantagens como indicador de qualidade do solo.

As plantas espontâneas também foram comentadas, e a respeito da sua existência, foi constatado que quanto maior a sua diversidade, maior é o equilíbrio do meio, pois desordens químicas ou físicas no solo favorecem um número restrito de espécies.

Em todas as capacitações, os pontos mais abordados pelos agricultores foram aqueles relacionados à qualidade do solo, o que demonstra o entendimento das características do solo benéficas às suas funções e que estão diretamente ligadas a produção. Quanto à sustentabilidade, a abordagem foi bem menor, evidenciando uma deficiência no conhecimento a respeito da produção sustentável e seus benefícios. 


\section{CONSIDERAÇÕES FINAIS}

As capacitações e testes propostos aos agricultores foram muito importantes para fazê-los perceber os fatores que envolvem toda a cadeira produtiva de uma propriedade e que esses fatores interagem entre si e são determinantes no equilíbrio do sistema. Desta forma são decisivos no incremento e manutenção da qualidade do solo e na busca pela sustentabilidade.

Também permitiu aos agricultores medir a condição atual do solo e todo o sistema envolvido. Assim, poderão perceber a evolução na qualidade da produção e adotar os melhores métodos de produção e conservação.

Enfim, foi possível construir com os agricultores, com o auxílio de professores e técnicos, um modo fácil e rápido de avaliação dos sistemas produtivos. As ações do projeto incentivam o produtor rural a desenvolver sua capacidade crítica e enxergar diferentes caminhos na busca de qualidade de vida e desenvolvimento sustentável, enquanto os estudantes envolvidos adquiriram experiência com ações participativas em extensão rural.

\section{AGRADECIMENTOS}

À Fundação CS-Fund/Warsh-Mott Legacy (Estados Unidos) pelo apoio financeiro, ao apoio da Empresa de Pesquisa Agropecuária e Extensão Rural de Santa Catarina - EPAGRI e de diversas Organizações Não Governamentais (ONGs) e demais participantes do projeto: Elisa Vilvert, Henrique von Hertwig Bittencourt, Ivan Bonjorno, Lauro Artur Otavio Martins, Marcos Alberto Lana.

\section{REFERÊNCIAS}

BITTENCOURT, H. H. Ecologia fitoquímica: entendo e manejando a competição entre vegetais em sistemas de plantas cultivadas. Florianópolis, 2007. Trabalho de Pós Graduação (Disciplina de Seminários) - Curso de Agronomia, Centro de Ciências Agrárias, Universidade Federal de Santa Catarina.

DOYLE, C.J. A review of the use of models of weed control in Integrated Crop Protection. Agriculture, Ecosystems and Environment, v. 64, p. 165-172, 1997.

DORAN, J. W.; PARKIN, T. B. Defining and assessing soil quality. In: DORAN, J. W.; COLEMAN, D. C.; BEZDICEK, D. F.; STEWARD, B. A. eds. Defining soil quality for a sustainable environment. Soil Science of America Special Publication n. 35, Madison, Wisconsin. P. 3-21, 1994. 
GARCIA, K. A. A percepção de qualidade do solo por agricultores através da aplicação da metodologia dos indicadores. Florianópolis, 2007. 35f. Monografia (Graduação em Agronomia) - Centro de Ciências Agrárias, Universidade Federal de Santa Catarina.

KOHLI, R. K.; BATISH, D. R.; e SINGH, H. P. Allelopathic interactions in agroecosystems. In: REIGOSA M.; PEDROL N. e L. GONZALEZ (Ed.). Allelopathy: A physiological process with ecological implications. Dordrecht: Springer, p. 465-492, 2006.

NICHOLLS, Clara Inês; ALTIERI, Miguel A. Sistema agroecologico rápido de evaluación de calidad de suelo y salud de cultivos en el agroecosistema de café. Universidad de California, Berkeley. 2004.

STOCKING, M. A; MURNAGHAN, N. Handbook for the field assessment of land degradation. Earthscan Publications, London. 2001.

VALLE, L. E. de R.\& COSTA, R. B. da. Conservação ambiental e desenvolvimento local: a percepção dos produtores rurais. Disponível em $<$ Erro! A referência de hiperlink não é válida.> Acesso em: 22 fev. 2008.

ZILLI, J. E. et al. Diversidade microbiana como indicador de qualidade do solo. Cadernos de Ciência \& Tecnologia, Brasília, v. 20, n. 3, p. 391-411, set./dez. 2003. Disponível em <http://atlas.sct.embrapa.br/pdf/cct/v20/v20n3_01.pdf> Acesso em: 20 fev. 2008. 